\title{
INJECTION RELATED BACKGROUND DUE TO THE TRANSVERSE FEEDBACK*
}

\author{
F.-J. Decker, R. Akre, A. Fisher, R.H. Iverson, M. Weaver, SLAC, Stanford, CA 94309, USA
}

\section{Abstract}

The background in the BaBar detector is especially high during injection, when most components are actually having reduced voltages. The situation is worse for the beam in High Energy Ring (HER) when the LER beam is present. It was found that the transverse feedback system plays an important role when stacking more charge on top of existing bunches. Lowering the feedback gain helped and it was realized later that the best scenario would be to gate off the feedback for only the one bunch, which got additional charge injected into it. The explanation is that the blown-up, but centered, original HER bunch plus the small injected off-axis bunch (each with half the charge) would stay in the ring if not touched, but the feedback system sees half the offset and wants to correct it, therefore disturbing and scraping the blown-up part.

\section{INTRODUCTION}

The background in BABAR from PEP-II can have many different reasons. Offsets in the six phase space dimensions come to mind first $(x, x, y, y, E, \varphi)$. These are generally tuned for best injection rate and low background. The next order (matching) and other not anticipated effects are described in this paper.

\section{LATTICE MISMATCHES}

\subsection{Injected Beam}

Increasing the beam size at the injection septum by changing the betatron function from $35 \mathrm{~m}$ to $70 \mathrm{~m}$, the very high betatron region near the interaction point (IP) was reduced from $2400 \mathrm{~m}$ in Fig. 1 to $1200 \mathrm{~m}$. This helped to reduce injection background.

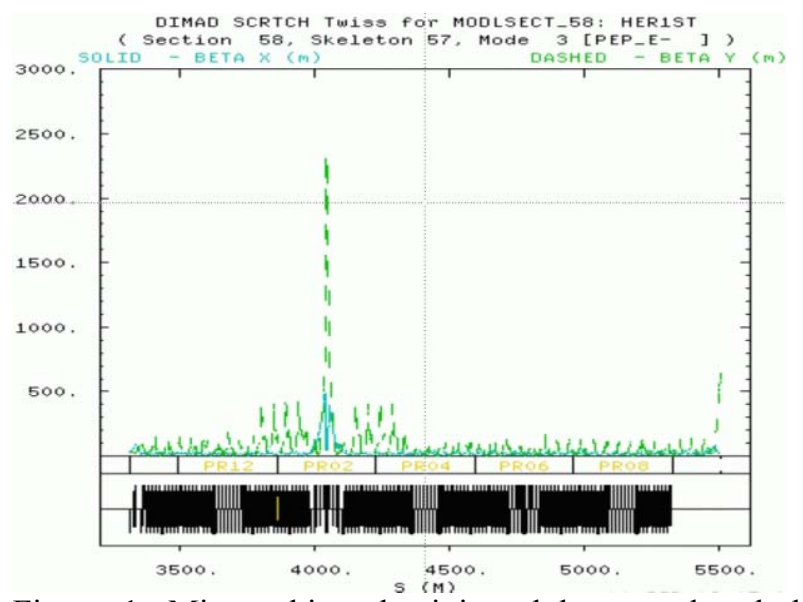

Figure 1: Mismatching the injected beam reduced the high betatron region near the IP $(z=4000 \mathrm{~m})$ into half.

\footnotetext{
*Work supported by Department of Energy contract DE-AC03-76SF00515.
}

\subsection{Mismatch between Injection Bump Kickers}

For injection a few hundred ns long part of the stored beam is kicked nearly $20 \mathrm{~mm}$, so close to the septum that the lifetime starts to degrade. If the kickers have different strengths or the phase advance between them is not $180^{\circ}$, the bump is not closed and an oscillation results. Figure 2 shows the stored beam orbit at the septum. The beam position monitor (BPM) indicates a $16 \mathrm{~mm}$ bump, but then in the following turns the beam oscillates by about $\pm 1 \mathrm{~mm}$. This is about only $6 \%$ of the bump, but two times the beam size in $y$.

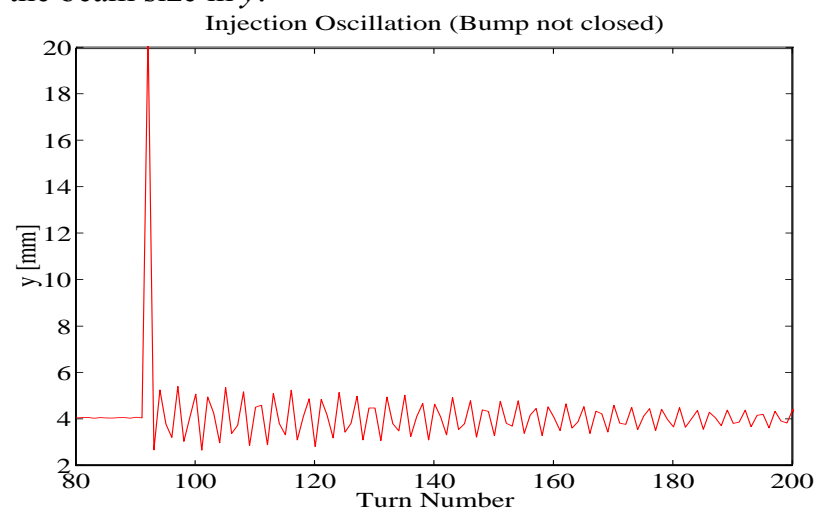

Figure 2: Injection oscillation due to a not perfectly closed bump.

To figure out whether it is the kicker strength or the phase advance in between, the first oscillation is plotted versus $z$ or BPM number in Fig. 3. The offset at the kicker is caused by about $11^{\circ}$ less phase advance than $180^{\circ}$.

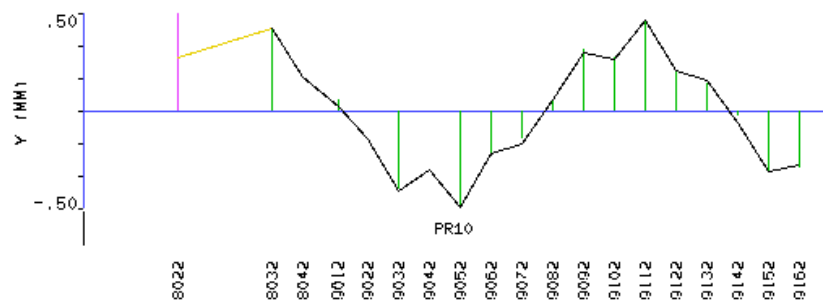

Figure 3: Oscillation due to non-closed bump, indicating offset at kicker (near BPM 8032). The septum is to the left, around it BPMs read 5.7, 16.7 and $6.7 \mathrm{~mm}$.

\section{BACKGROUND SIGNALS}

\subsection{Top Offs}

A top off into PEP-II can be as fast as 3 min (Fig. 5). Besides the injection rate, background signals from SVTdiodes are typically monitored. They roughly distinguish the induced background coming mainly from HER or LER. To get more information other signals like from the calorimeter (EMC) and trigger rate are monitored. 


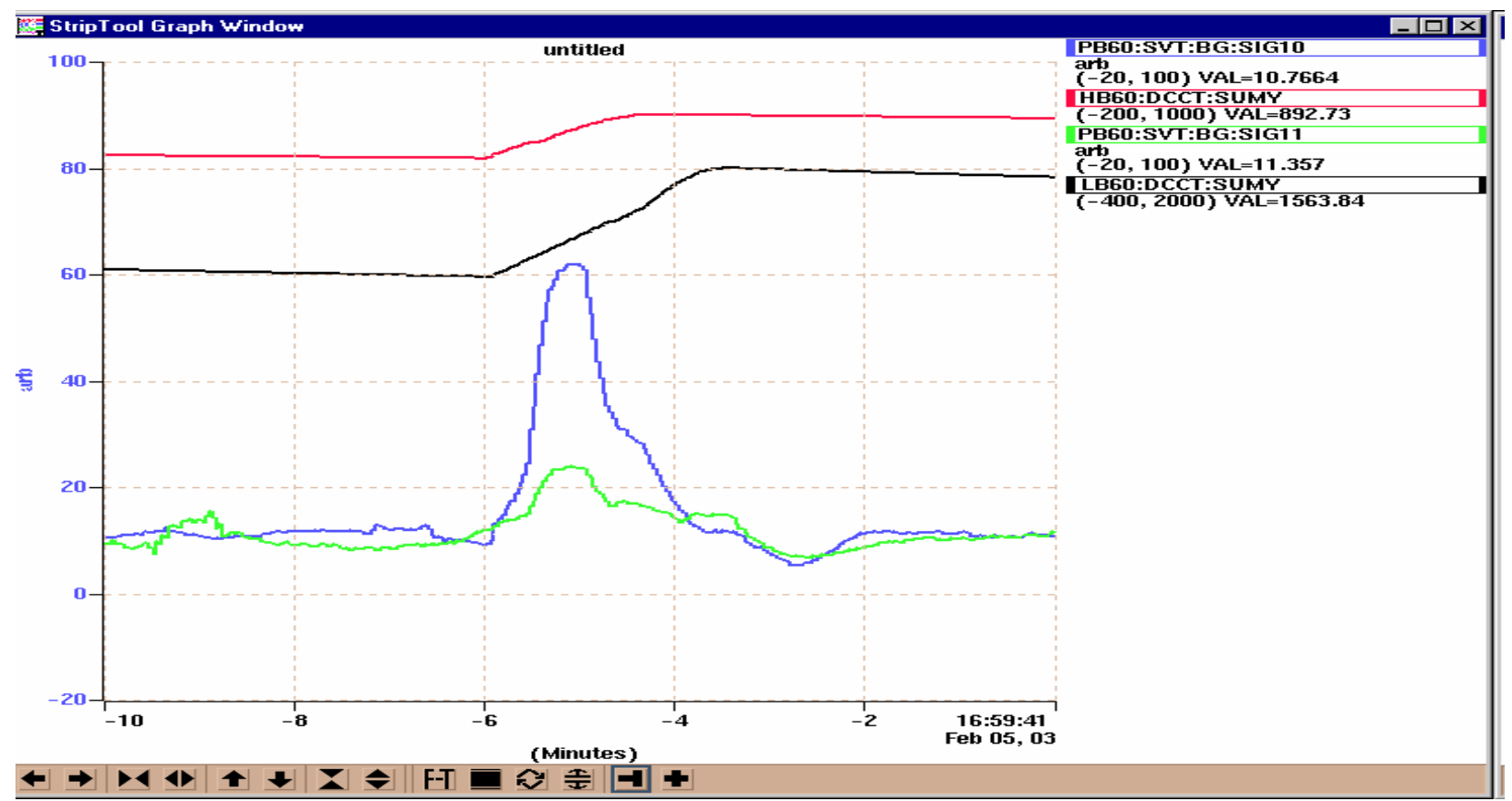

Figure 4: Three minutes top off injection. The top two lines show the HER and LER currents, then background signals SIG10 for HER and SIG11 for LER.

\subsection{Electromagnetic Calorimeter}

The BaBar electromagnetic calorimeter provides detailed information on backgrounds during injection. This detector consists of 6580 Th-doped CsI crystals, each readout by a pair of photodiodes. The photodiode response is amplified, shaped, and digitized at $3.72 \mathrm{MHz}$. The resulting readout stream is split onto two paths, one path to the BaBar trigger electronics and another to an intermediate buffer in the calorimeter readout boards where up to 256 samples $(68.8 \mu \mathrm{s})$ can be processed after a trigger decision. The BaBar trigger system generates trigger decisions from input on the first path, and a timestamp is recorded from a counter based on the PEP-II $476 \mathrm{MHz}$ clock divided by 8 .

The single-cluster trigger times $(\mathrm{E}>100 \mathrm{MeV})$ are compared with the times of individual injection shots. This data source has small deadtime $(2.7 \mu$ s per trigger $)$ and runs in parallel to the normal BaBar data acquisition. On the other hand, during periods of abnormally high backgrounds in HER injection the trigger system can suffer inefficiency from detector saturation effects.

An example of background trigger timing with respect to HER injection is shown in Fig. 5. Background trigger rates are typically heightened for the first $30 \mu$ s after injection and then rise again over the next $3-5 \mathrm{~ms}$. The trigger rates fall over a time scale of roughly $5 \mathrm{~ms}$. Finer analysis of the timing confirms that the additional triggers occur at intervals of $7.34 \mu \mathrm{s}$, the revolution period of the injected bunch. The injection backgrounds also depend upon the progress into a fill. Figure 6 displays the injection backgrounds for both the LER and HER when filling from 0 to maximum current. A Fourier transform of the background trigger timing, also in Fig. 6, shows a distinct contribution from synchrotron oscillations. The observed HER synchrotron oscillation frequency drift could be from time-lacking of the HER RF cavity tuners. HER_NumTrigs50

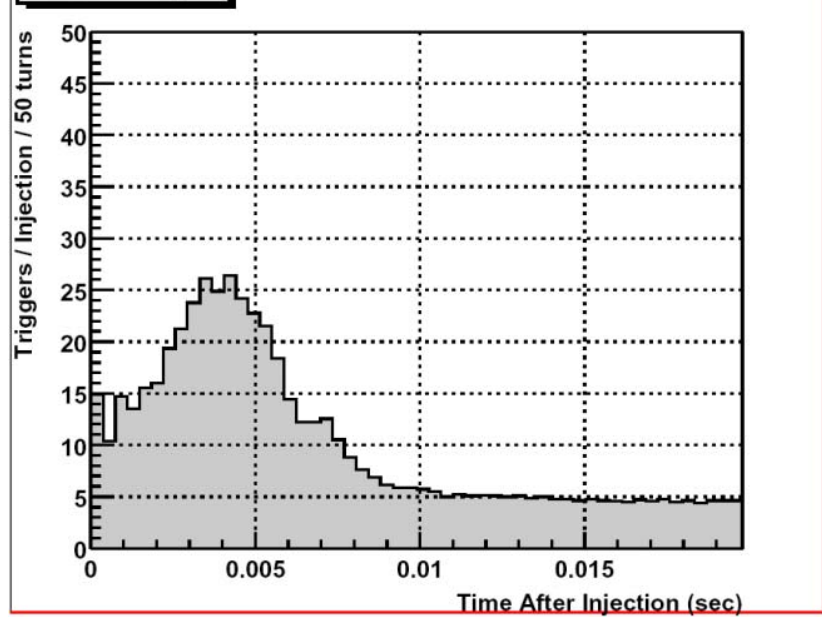

Figure 5: Calorimeter triggers show a large background increase several $\mathrm{ms}$ after an injection.

The second calorimeter readout path can be used to obtain unbiased sampling at well-defined times using the PEP injection timing signal and a programmable delay generator. This data includes the detector response magnitude and its sampling is immune to detector effects associated with high backgrounds. Figure 7 shows an example of the calorimeter energy measurements accumulated over several scans of the $7 \mathrm{~ms}$ period following an injection. The LER synchrotron oscillation component is observed to be a greater exposure contribution than indicated by the trigger analysis. 


\section{LER}

HER

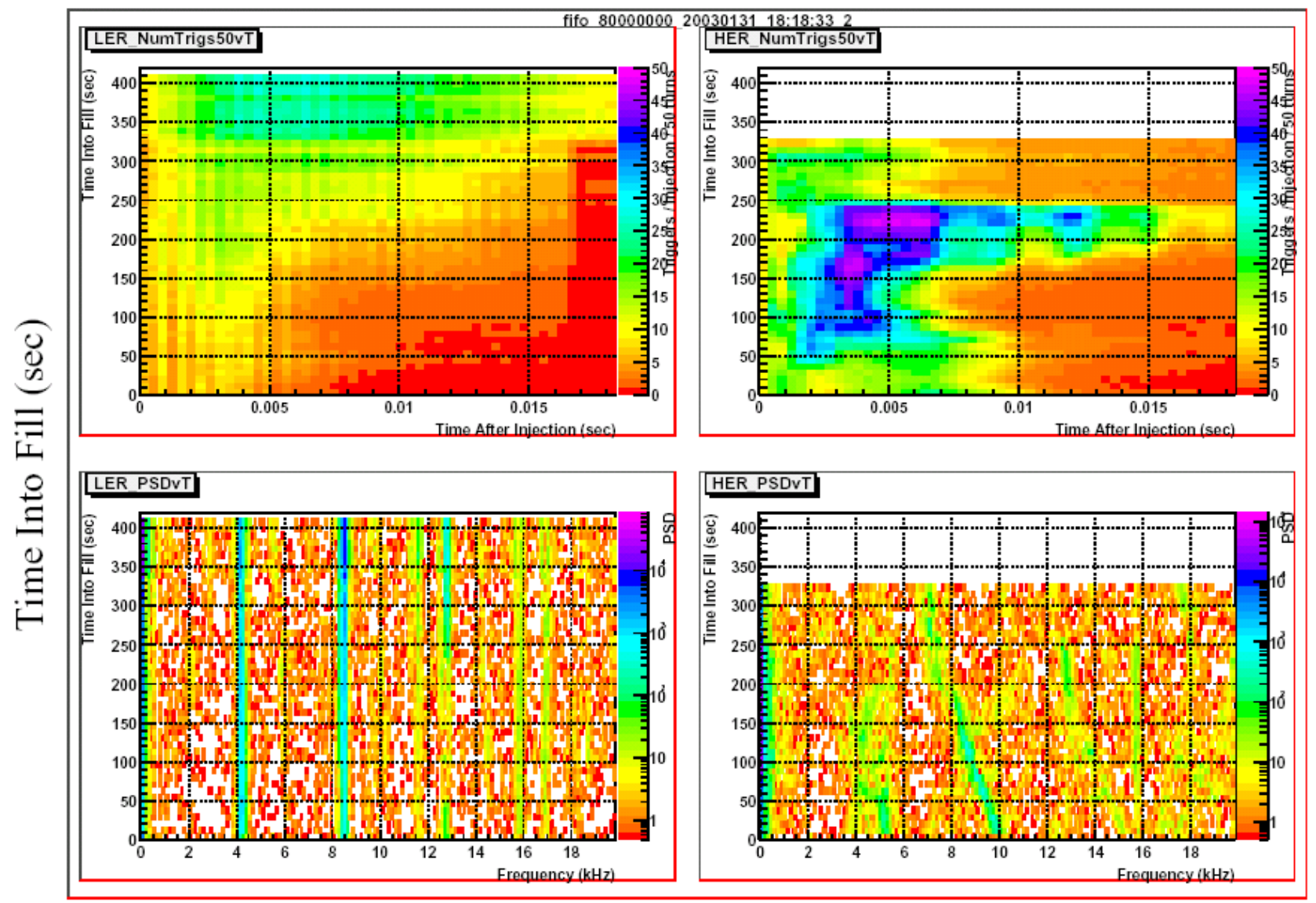

Figure 6: Injection backgrounds increase as a fill progresses. The period from 0 to 240 seconds consists of large charge quanta injection into the HER and LER at $15 \mathrm{~Hz}$ each. The period from 240 to 320 seconds uses small charge quanta injection into the HER. The period from 320 to 410 seconds includes $30 \mathrm{~Hz}$ injection into the LER.

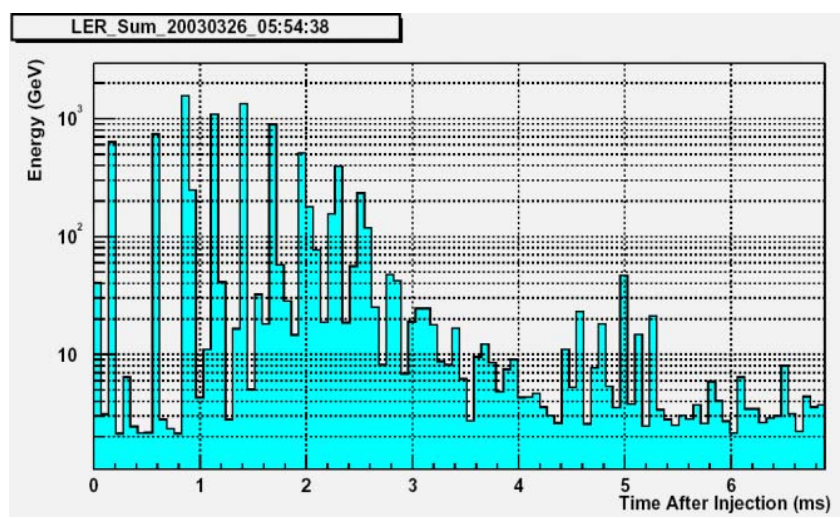

Figure 7: Calorimeter background energy measurements during the $7 \mathrm{~ms}$ following an injection. The energy scale is averaged per injection.

\section{POSSIBLE FIXES}

First offsets and sizes $\left(1^{\text {st }}\right.$ and $2^{\text {nd }}$ order effects $)$ need to be tuned. Speeding up the HER low level RF feedback system, which controls the tuners, should eliminate the shift of the synchrotron tune lines. The tricky part is the long lasting $(>5 \mathrm{~ms})$ background from Fig. 5, which is faster than the damping time $(40 \mathrm{~ms})$. Since the observed effect with the fast transverse feedback system (described in the abstract), we are planning to gate off the feedback only for the bunch with additional injected beam for about $30 \mathrm{~ms}$. The current set up should be able to do this up to $1 \mathrm{~ms}$ which seems too short. The $30 \mathrm{~ms}$ fits good compared with a $30 \mathrm{~Hz}$ injection (= $33.33 \mathrm{~ms}$ ), so we plan to have no feedback on only one bunch at a time and hope that the injected beam is filamented enough that it does not represent any offset, which the feedback wants to fix.

\section{REFERENCES}

[1] R.L. Holtzapple,et al., "Observation of Beam Size Flip-Flop in PEP-II", EPAC02, Paris, June 2002.

[2] F.-J. Decker et al., "Increasing the number of Bunches in PEP-II", EPAC02, Paris, June 2002. 